\title{
Evaluation of Two Programs Developed for Dental Digital Subtraction Radiography: a Comparison between Different Methods of Geometric Alignment
}

\author{
Marcin Kozakiewicz $^{1}$, Katarzyna Bogusiak ${ }^{1}$, Marcin Denkowski ${ }^{3}$, Marcin Hanclik $^{2}$ \\ ${ }^{1}$ Clinical Department of Maxillofacial Surgery \\ Medical University of Łódź, Military Teaching Hospital, Veterans Central Hospital \\ ul. Żeromskiego 113, 91-647 Łódź, Poland \\ e-mail:marcin.kozakiewicz@umed.lodz.pl;katarzyna.bogusiak@gmail.com; \\ ${ }^{2}$ Department of Cranio-Maxillofacial and Oncological Surgery, Medical University of Łódź \\ University Hospital Nr. 1 \\ ul. Kopcińskiego 22, 90-153 Łódź, Poland \\ e-mail:mhanclik@googlemail.com \\ ${ }^{3}$ Department of Information Technologies, University of Maria Curie-Skłodowska, \\ Pl. M. Curie-Skłodowskiej 5, 20-031 Lublin, Poland \\ e-mail:denmar@goblin.umcs.lublin.pl
}

(Received: 23 December 2008; revised: 22 August 2009; accepted: 16 September 2009; published online: 29 November 2009)

\begin{abstract}
Purpose: The aim of this research was to compare different methods of geometric alignment produced by two programs developed to be utilized for digital subtraction radiography (DSR). Material and Methods: Material consists of 50 pairs of intra-oral radiographs taken in 50 patients during normal oral clinical treatment. Two programs invented by the authors of this article: ToothVis 1.4 (TV) and DentalStudio 2.0 (DS) software were used in this study. Images obtained by two methods of DSR (cut and divide) and geometrically aligned with four methods were compared with the use of the peak signal to noise ratio (PSNR). Results: Analyzing the PSNR, it was observed that for cut subtraction its values ranged from $37.93 \mathrm{~dB}$ to $39.99 \mathrm{~dB}$. For divide subtraction the PSNR values varied between $39.00 \mathrm{~dB}(03$ pt_DS vs 10 pt_TV) and $47.73 \mathrm{~dB}$ (03_pt TV vs 10 pt_TV). The PSNR achieved higher values for divide than for cut subtraction. Comparing cut and divide subtraction, the PSNR was the lowest for 3-point geometric alignment. Conclusion: Geometric alignment with the ToothVis software combined with divide subtraction gives the best quality of a subtracted image.
\end{abstract}

Key words: digital radiography, technical aspects, experimental investigations, computer applications - general, efficacy studies

\section{INTRODUCTION}

Since Ziedses des Plantes introduced subtraction methods in 1930s, this so-called first generation of subtraction systems employed photographic techniques for the subtraction of a priori registered radiographic films that were aligned manually, and new generations of subtraction systems have been invented subsequently [1]. Although the digital subtraction radiography (DSR) is not the latest advancement in dental imaging, and the interest in DSR has diminished over the last couple of years, it is still a good and cheap imaging method in contrary to Cone Beam CT technology. It offers some distinct advantages over film, but also presents different challenges to overcome. With the use of DSR, a meaningful comparison between two images of the same object can be made on condition that they have the same projection geometry and the same contrast $[2,3]$. A number of devices have been invented for serial recording of intraoral radiographs, very often consisting of complex mechanical methods based on bite impressions and fixation between the patient, the film holder, and an X-ray tube [4-9]. However, discrepancies between the subtracted images still occur. They are related to different projection geometry, mismatches in X-ray tube settings, density differences due to film development, digitization procedures, and precision of superimposition of unchanged anatomical features in the scanned images $[2,3]$. New programs are being invented to provide sufficient geometric uniformity of subsequent radiographs, and to eliminate discrepancies in grey levels between the images 
$[1,10]$. Still, new approaches are needed to provide better geometric alignment as well as brightness and contrast adjustment between the subtracted images.

The aim of this research was to compare different methods of geometric alignment produced by two programs developed to be utilized for DSR.

\section{METHODS}

Material consists of 50 pairs of intra-oral radiographs taken of 50 patients during normal clinical treatment (Fig. 1). The Digora Optime system of digital intra-oral radiography (Soredex, Tuusula, Finland) was applied in this study. Radiographs were taken in a standardized way [11]. The same radiological apparatus was used for all radiographs: the Focus X-ray intraoral unit (Instrumentarium Dental, Tuusula, Finland). Technical parameters of exposition were the same in all included radiographs: $7 \mathrm{~mA}, 70 \mathrm{kV}$, and $0.06 \mathrm{~s}$. The time interval between radiographs performance was three months.

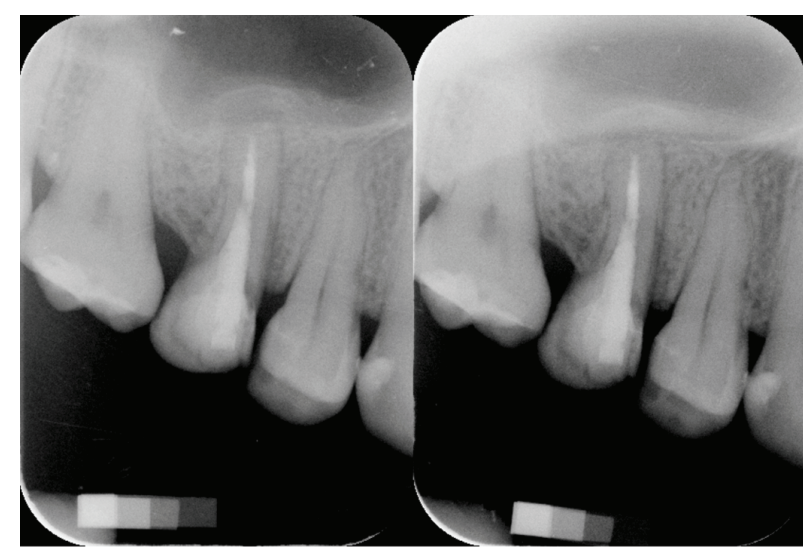

Fig. 1. A pair of intra-oral radiographs recorded with slightly different angulations

Our research is based on two programs invented by the authors of this article: ToothVis 1.4 (TV) and DentalStudio 2.0 (DS) software. Our algorithms applied in both programs perform automated contrast correction and manual geometric alignment. Both programs use at least 3 manually positioned reference points for any manipulation of radiographic images, and for TV the number of maximal points is unlimited.

\section{Dental Studio geometric-alignment algorithm}

This registration procedure is based on finding such 2-dimensional affine transformations, considered globally, that directly transform the control points in the fixed image (baselineradiograph) to the control points in the moving image (radiograph taken 3 months later) [12]. This algorithm provides exact affine transformation of the control points, i.e. after transformation the control points on the moving image have exactly the same positions as on the fixed image.

\section{Toothvis geometric-alignment algorithm}

The idea of this registration method aims at finding such 2-dimensional transformations, considered globally, that minimize the Euclidean distance between corresponding control points between the fixed and the moving image [12]. To minimize the selected similarity function, the Powell optimization algorithm was applied [13].

Powell's method of optimization is an iterative algorithm which optimizes the n-dimensional vector of parameters for a one-valued minimization function. In other words, in subsequent iterations, this method searches for such elements of the vector to which a given minimization function has a minimum value. In our case, the Euclidean distance between corresponding control points between the fixed and the moving image is taken as the minimization function, and the elements of the vector being optimized are parameters of a 2-dimensional affine transformation. This transformation consists of translations and both rotations and scalings applied relatively to the center of a transformed image.

This algorithm provides approximate transformation of the control points, i.e. after transformation the control points on the moving image do not usually have the same positions as on the fixed image.

Fifty pairs of digital intraoral radiographs were geometrically corrected in these two programs. There was one person who acquired the images, and one operator who manually positioned the reference points. Post-process geometric standardization in both programs is based on locating landmarks as reference points. In each of the 50 pairs of images, three reference points were manually positioned for both programs and, additionally, two further methods of geometric alignment of four and ten points were applied for TV. For three-point alignment the same anatomical structures were chosen for both programs. Four-point and ten-point alignment enclosed the same landmark as in three-point alignment.

In all radiographs the same metal pattern was embedded, i.e. the metal plate with areas of various thickness. This enables a comparison of the grey levels among all radiographs neutralizing the influence of exposure differences. The procedure is as follows:

On each pair of fixed and moving images the areas of highest and lowest metal thickness (the lowest and highest 
grey level on those areas, respectively) were marked. The grey scales on both images were assumed to be linear. The computed linear function from the fixed image was further applied to the moving image, thus enabling to match the grey levels in those radiographs.

After the grey scale adjustment was performed in DS, as the specialized software for digital radiological subtraction, two methods of digital subtraction were performed: "cut" and "divide" in four series of separately aligned pairs of radiographs (Fig. 2). "Cut" subtraction means erasing marginal data (included in 256 grey levels) from 9-bit meta-image constructed by the DS during application of a subtraction algorithm. In those marginal areas of histogram there were no real data. "Divide" subtraction means erasing every second level (the number of all grey levels were divided by two) from 9-bit meta-image constructed by the DS during application of the subtraction algorithm. Eventually, thanks to this procedure the operator received a standard 8-bit subtraction image.

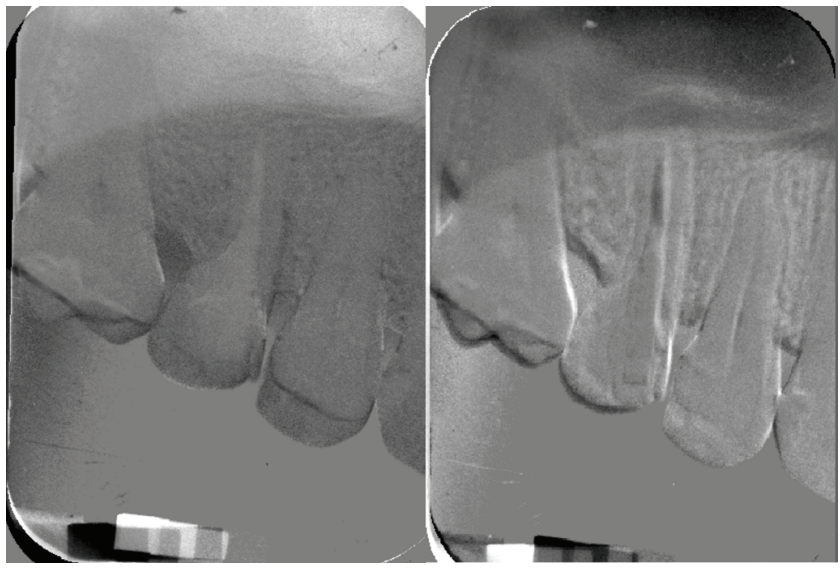

Fig. 2. A comparison of two subtracted images aligned by two different programs: DS (on the left side) and TV (on the right side)

Then, the ROI (region of interest, area of $100 \times 100$ pixels located among landmarks) was drawn in all 400 subtraction images (100 corrected geometry with DS, 300 corrected geometry with TV). Regions of interest were selected in images of non-treated and non-pathological areas in the radiographs. They were located in areas of the best geometrical adjustment. The arithmetical mean from the brightness value of each pixel of ROI was performed. Subsequently, to compare images obtained by two methods of digital subtraction radiography ("cut" vs. "divide") and geometrically aligned with four methods, the peak signal to noise ratio (PSNR) calculation, a well-known criterion that is commonly used to assess the quality of reconstructed images, was performed: ${ }^{14}$

$$
P S N R=20 \log _{10} \frac{2^{d}-1}{\left[\frac{1}{n} \sum_{k=1}^{n}\left(i_{k}-j_{k}\right)^{2}\right]^{\frac{1}{2}}}
$$

where $d$ is the colour depth, $i_{k}$ and $j_{k}$ are the pixel intensities at position $k$ of the test and the modified images, respectively, and $\mathrm{n}$ is the total number of pixels in the image [13].

\section{Statistical analysis}

Mean and medians were compared in Statgraphics Plus for Windows ver. 5.1. For normal distribution a t-test, and for other distributions the W-test, were done. Significance was indicated for $p<0.05$.

\section{RESULTS}

Comparing the mean scale of grey of ROI, it was observed that the "cut" subtraction presented a larger variability level than the "divide" subtraction. The smallest variability was noticed after 10-point alignment and subtracted with the "divide" method, while the largest - after 3-point alignment. It was larger for images normalized with DS than with TV (after 3-point alignment). The median grey level for the "cut" subtraction presented a lower grey level value than for the "divide" subtraction considering 3-point alignment in both programs DS and TV. Taking into consideration other methods of geometric alignment, the opposite tendency for "cut" and "divide" subtractions was observed (Fig. 3). There are significant differences $(p<0.05)$ in mean optical density among subtraction methods and geometric alignment schedule type procedure and among geometric alignment methods within one subtraction method (Table 1).

Table 1. Mean optical density of subtraction image [ROI $100 \times 100$ pixels]

\begin{tabular}{l|c|c|c|c}
\hline & 03_pt_DS & $03 \_p t \_T V$ & $04 \_p t \_T V$ & $10 \_p t \_T V$ \\
\hline CUT & $126.87 \pm 1.98$ & $125.86 \pm 1.87$ & $128.04 \pm 1.65$ & $126.57 \pm 1.80$ \\
\hline DIVIDE & $128.71 \pm 0.99$ & $126.57 \pm 0.87$ & $126.95 \pm 0.68$ & $126.09 \pm 0.53$ \\
\hline
\end{tabular}

Legend: There are significant differences for $p<0.05$ among cut and divide subtraction methods according to the geometrical normalization method, respectively [columns], and among geometrical normalization method within one subtraction method [rows]. For normal distribution the $t$-test, and for other distributions the W-test, were done (all 3-point alignment method results had other than normal distributions).

CUT - cut subtraction method; DIV - divide subtraction method; 03 pt three-point geometric alignment method; 04 pt - four-point geometric alignment method; $10 \_$pt - ten-point geometric alignment method; DS DentalStudio alignment software; TV - ToothVis alignment software 

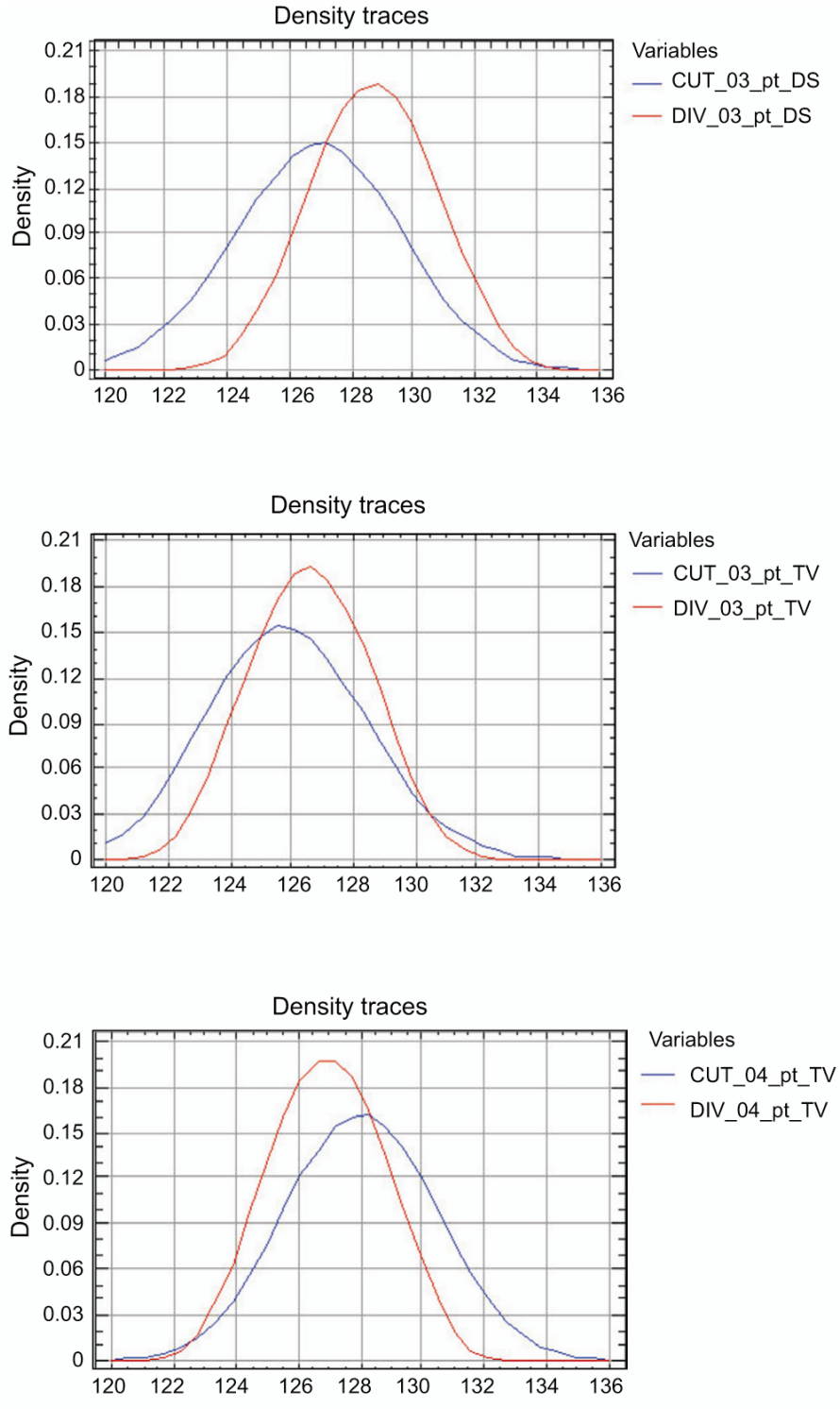
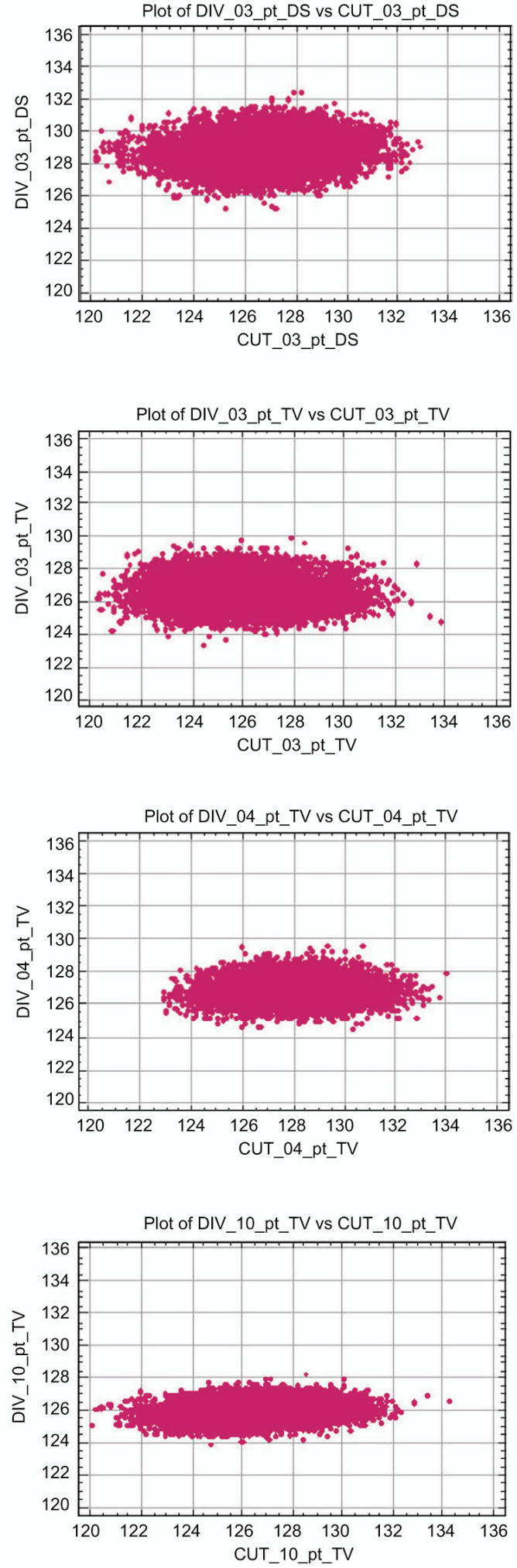

Fig. 3. Observed values as shade of grey in ROI. On the left side there are density traces and on the right side - a plot of completed data

Legend: CUT - cut subtraction method; DIV - divide subtraction method; 03 pt - three-point geometric alignment method; 04 pt - four-point geometric alignment method; $10 \mathrm{pt}$ - ten-point geometric alignment method; DS - DentalStudio alignment software; TV - ToothVis alignment software 
image performance, as PSNR is a primary determiner of perceptibility in an image. Some distortions that occur in case of ROI alignment (in contrary to the whole image alignment) can be distributed throughout the image in different fashions. Values for PSNR range from infinity for identical images to 0 for images that have no commonality [16]. As expected, PSNR increases along with the similarity of the modified image to the reference image. For a grey scale image with $\mathrm{d}$ bits per pixel, the typical PSNR values shall range between 20 and 40 [10].

Three-point geometric alignment in TV is a less efficient method than 3-point alignment in DS [Table 2: 03_pt_TV vs 04_pt_TV $=37.94 ; 03 \_p t \_D S$ vs 04_pt_TV=39.99], especially as we assume that generally the 3-point alignment is less efficient than the 4 or 10-point ones [11]. Unlike the "divide" subtraction, 3-point alignment in DS [03_pt_DS vs $10 \_p t$ TV $\left.=39.00\right]$ is worse than 3-point alignment in TV [03_pt_TV vs 10_pt_TV=47.74].

Table 2. PSNR when different methods of geometric alignment are compared

\begin{tabular}{|c|c|c|}
\hline & Cut subtraction & Divide subtraction \\
\hline $\begin{array}{l}\text { 03_pt_TV vs } \\
04 \_p t \text { tTV }\end{array}$ & 37.93 & 47.43 \\
\hline $\begin{array}{l}\text { 03_pt_TV vs } \\
10 \text { pt_TV }\end{array}$ & 39.48 & 47.73 \\
\hline $\begin{array}{l}04 \_p t \_T V \text { vs } \\
10 \_p t \_T V\end{array}$ & 39.24 & 46.31 \\
\hline $\begin{array}{l}03 \text { pt_DS vs } \\
03 \_ \text {pt_TV }\end{array}$ & 39.00 & 40.21 \\
\hline $\begin{array}{l}03 \text { 03.pt_DS vs } \\
04 \_p t \text { TV }\end{array}$ & 39.99 & 41.78 \\
\hline $\begin{array}{l}03 \text { 03t_DS vs } \\
10 \_ \text {pt_TV }\end{array}$ & 39.07 & 39.00 \\
\hline
\end{tabular}

Legend: $03 \mathrm{pt}$ - three-point geometric alignment method; $04 \mathrm{pt}$ - fourpoint geometric alignment method; $10 \_$pt - ten-point geometric alignment method; DS - DentalStudio alignment software; TV - ToothVis alignment software

The general trend towards higher PSNR values in the "divide" images indicates a lower noise ratio in comparison to the "cut" images. The differences between the PSNR values in the "cut" subtraction were comparatively small. The "cut" subtraction produces more rough images than the "divide" subtraction because of enhances in grey level variability by augmentation of central grey levels from 9-bit meta-image during the process of construction of the final 8-bit subtraction image. The difference (lower value of PSNR) between methods of subtraction performance are gradually eliminated (higher value of PSNR) when a higher number of points is used in geometric alignments (Fig. 4).

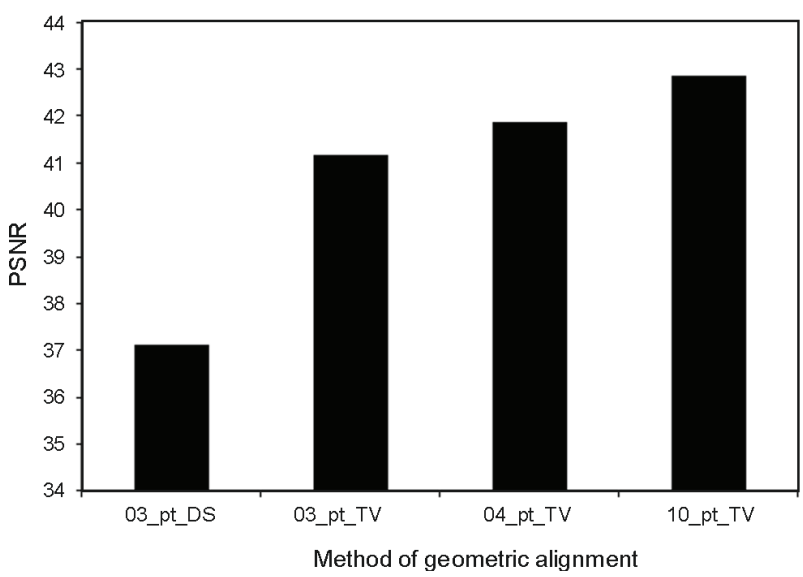

Fig. 4. PSNR values when cut versus divide subtraction images are compared

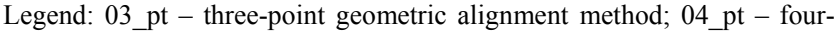
point geometric alignment method; $10 \_$pt - ten-point geometric alignment method; DS - DentalStudio alignment software; TV - ToothVis alignment software

This relation is dependent on the method of geometric alignment. A more detailed method of elimination of the geometric deformation triggers a lower noise ratio in the subtracted images (higher PSNR values). The highest PSNR values were observed in divide subtraction for images geometrically aligned with TV. Therefore, this method of geometric standardization and contrast correction is less noisy than others compared in this study.

In conclusion, geometric alignment with the ToothVis software combined with the "divide" subtraction gives the best quality of the subtracted image.

\section{Acknowledgements}

The study was supported by the Medical University of Łódź grant nr 502-12-604.
Abbreviations:
CUT - cut subtraction
DIV - divide subtraction
DS - DentalStudio
PSNR - peak signal to noise ratio
$\mathrm{TV}$ - ToothVis 


\section{References}

[1] T.M. Lehmann, H.G. Gröndahl and D.K. Benn, Computer-based registration for digital subtraction in dental radiology. Dentomaxillofac Radiol. 29, 323-346 (2000).

[2] J. Samarabandu, K.M. Allen, E. Hausemann and R. Acharya, Algorithm for the automated alignment of radiographs for image subtraction. Oral Surg Oral Med Oral Pathol Oral Radiol Endod 77, 75-79 (1994).

[3] D.C. Yoon, A new method for the automated alignment of dental radiographs for digital subtraction radiography. Dentomaxillofac Radiol 29, 11-19 (2000).

[4] D.J. Rudolph and S.C. White, Filmholding instrument for intraoral subtraction radiography. Oral Surg Oral Med Oral Pathol 65, 767-772 (1988).

[5] E.B. Nery, J.W. Olson, J.M. Henkin and J.H. Kalbfleish, Filmholder device for radiographic assessment of periodontal tissues. J Periodont Res 20, 97-105 (1985).

[6] L.C. Carpio, E. Haosmann, R.G. Dunford, K.M. Allen and L.A. Christersson, Evaluation of a simple modified radiograph alignment system for routine use. J Periodontol 65, 62-67 (1994).

[7] I. Sewerin, Device for serial intraoral radiography with controlled projection angels. Dan Dent J 94, 613-616 (1990).

[8] A. Wenzel and I. Sewerin, Sources of noise in digital radiography. Oral Surg Oral Med Oral Pathol 71, 503508 (1991).
[9] U. Zappa, C. Simona, H. Graf and J. van Aken, In vivo determination of radiographic projection errors produced by a novel filmholder and $x$-ray beam manipulator. J Periodontol 62, 674-683 (1991).

[10] P. Güneri, S. Goğüş, Z. Tuğsel, A. Ozturk, C. Gungor, and H. Boyacioğlu, Clinical efficacy of a new software developed for dental digital subtraction radiography. Dentomaxillofac Radiol 35, 417-421 (2006).

[11] M. Kozakiewicz, K. Bogusiak, M. Hanclik, M. Denkowski and P. Arkuszewski, Noise in subtraction images made from pairs of intraoral radiographs: a comparison between four methods of geometric alignment. Dentomaxillofac Radiol 37, 1-7 (2008).

[12] T.M. Lehmann, H.G. Gröndahl and D.K. Benn, Review Article. Computer-based registration for digital substraction in dental radiology. Dentomaxillofac Radiol 29, 323-346 (2000).

[13] A. Saul, W. T. Teukolsky and B.P. Vetterling, Flannery. Numerical Recipes in $\mathrm{C}++$. In: William H., editor. Numerical Recipes in $\mathrm{C}++$ : The Art of Scientific Computing. Cambridge: Cambridge University Press; 2002, p. 412.

[14] D. Hearn and P. Baker, Computer graphics with open GL. 3 rd ed. New Jersey: Prentice Hall, 420-458 (2003).

[15] D. Solomon, Data compression. The complete reference. Berlin: Springer 240-242 (2000).

[16] M. Tur and E.L. Goldstein, Dependence of error rate on signal-to-noise ratio in fiber-optic communication systems with phase-induced intensity noise. Journal of Lightwave Technology 12, 2055-2058 (1989).
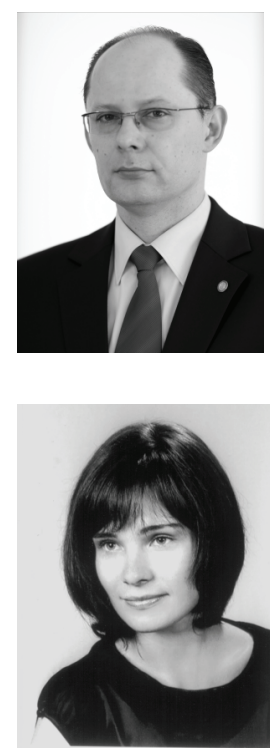

Marcin Kozakiewicz received the Ph. D. degree in 1996 and Dr. Hab. degree in 2005 from the Medical University of Łódź. Currently he is Chief of the Department of Maxillofacial Surgery at the Medical University of Łódź. He is specialinist of oral and maxillofacial surgery. His research interests focus on digital image analysis, oral and maxillofacial implantology, and telemedicine. Two journals from Philadelphia List engaged him as reviewer. He is author or co-author of 107 papers in major professional journals, 116 conference proceedings, and 5 patents.

Katarzyna BogusiaK graduaded from the Faculty of Medicine at the Medical University of Łódź in 2009. Her research interests concern digital image analysis in oral and maxillofacial implantology, and vascular surgery. She is author or co-author of 5 papers in major professional journals. 

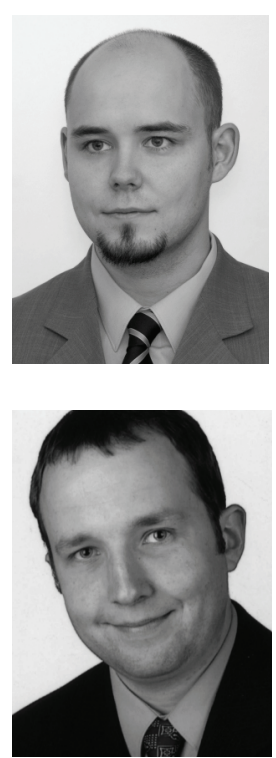

Marcin DenKowski received his M.Sc. degree in Physics from the Maria Curie-Skłodowska University in 2003. Currently he is a researcher with the Institute of Computer Science of the Maria CurieSkłodowska University. His research interests concern computer graphics and digital image processing, in particular with medical imaging and digital photography.

MarCIN Hanclik received his M. Sc. degree in Computer Science from the Warsaw University of Technology, and his M.A. degree in Economics from the Warsaw School of Economics, both in 2002. His research interests comprise software design, compiler construction, 3D modelling, biomechanics and application of the numerical methods in medicine. At present he is holding the position of Director of Engineering at ACCESS Systems Germany, a global provider of advanced software technologies to the mobile and beyond-PC markets, where he actively contributes to the evolution of the World Wide Web in $\mathrm{W} 3 \mathrm{C}$ and related standardization bodies. 